\title{
Bounds for double zeta-functions
}

\author{
ISAO KIUCHI AND YoshIO TANIGAWA
}

\begin{abstract}
In this paper we shall derive the order of magnitude for the double zeta-function of Euler-Zagier type in the region $0 \leq \Re s_{j}<1 \quad(j=1,2)$. First we prepare the Euler-Maclaurin summation formula in a suitable form for our purpose, and then we apply the theory of double exponential sums of van der Corput's type.
\end{abstract}

Mathematics Subject Classification (2000): 11L07 (primary); 11M41 (secondary).

\section{Introduction}

Let $s_{j}=\sigma_{j}+i t_{j} \quad(j=1,2, \ldots, r)$ be complex variables. The $r$-ple zeta-function of Euler-Zagier type is defined by

$$
\zeta_{r}\left(s_{1}, \ldots, s_{r}\right)=\sum_{1 \leq n_{1}<\cdots<n_{r}} \frac{1}{n_{1}^{s_{1}} \cdots n_{r}^{s_{r}}},
$$

which is absolutely convergent for $\sigma_{r}>1, \sigma_{r}+\sigma_{r-1}>2, \ldots, \sigma_{r}+\cdots+\sigma_{1}>r$. The function $\zeta_{r}$ has many applications to mathematical physics. In particular, algebraic relations among the values of $\zeta_{r}$ at positive integers have been studied extensively [14]. As a function of the complex variables $s_{j}$, the analytic continuation of $\zeta_{r}$ has been dealt already. For $r=2$, this problem was studied by F. V. Atkinson [3] in his research on the mean value formula of the Riemann zeta-function. For general $r$, the analytic continuation was proved by S. Akiyama, S. Egami and Y. Tanigawa [1] and J. Q. Zhao [17] independently, and later by K. Matsumoto [13]. The values at negative integers were considered in [2].

On the other hand, the order of magnitude of the zeta-function on some vertical line plays an important role in the theory of zeta-functions, e.g. it is used for the estimation of the sum of arithmetical functions (see below). The aim of this paper is to study such a problem for the double zeta-function of Euler-Zagier type:

$$
\zeta_{2}\left(s_{1}, s_{2}\right)=\sum_{1 \leq m<n} \frac{1}{m^{s_{1}} n^{s_{2}}} .
$$

Received June 9, 2006; accepted in revised form October 11, 2006. 
Before stating our results, we shall recall the previous result for the Riemann zeta-function $\zeta(s)$ and the double zeta-function $\zeta_{2}\left(s_{1}, s_{2}\right)$. Let $\mu(\sigma)$ denote the infimum of a number $c \geq 0$ such that

$$
\zeta(\sigma+i t) \ll|t|^{c},
$$

or alternatively as

$$
\mu(\sigma)=\limsup _{t \rightarrow \infty} \frac{\log |\zeta(\sigma+i t)|}{\log t} .
$$

As for a classical result for the function $\mu(\sigma)$ it is known that (see A. Ivić [11, Theorem 1.9])

$$
\mu(\sigma)= \begin{cases}\frac{1}{2}-\sigma & \text { if } \sigma \leq 0 \\ 0 & \text { if } \sigma \geq 1\end{cases}
$$

and

$$
\mu(\sigma) \leq \frac{1}{2}(1-\sigma) \quad \text { if } 0 \leq \sigma \leq 1 .
$$

Furthermore it is well known that

$$
\zeta(i t) \ll|t|^{\frac{1}{2}} \log |t|
$$

and

$$
\zeta(1+i t) \ll(\log |t|)^{\frac{2}{3}}
$$

for $|t| \rightarrow \infty$ (Ivić [11, p. 144 (6.7)]). In the case of $\sigma=\frac{1}{2}$, which is the most important in the theory of zeta-function, the first non-trivial result

$$
\mu\left(\frac{1}{2}\right) \leq \frac{1}{6}
$$

was obtained by G. H. Hardy and J. E. Littlewood (see [11]). The best estimate hitherto proved is $\mu\left(\frac{1}{2}\right) \leq \frac{89}{570}=0.156140 \ldots$ due to M. N. Huxley [8]. (He announced that he got an improvement $\mu\left(\frac{1}{2}\right) \leq \frac{32}{205}=0.156098 \ldots$ [9].)

Concerning the multiple zeta-function, $\mathrm{H}$. Ishikawa and K. Matsumoto used the Mellin-Barnes integral formula to obtain some results on the order of magnitude on the line $\sigma_{1}=\sigma_{2}=0$. In fact, they [10] showed that for a fixed $\alpha(\neq \pm 1)$ and any $\varepsilon>0$,

$$
\zeta_{2}(i t, i \alpha t) \ll(1+|t|)^{\frac{3}{2}+\varepsilon}
$$

and

$$
\zeta_{3}(-i t, i t, i t) \ll(1+|t|)^{\frac{5}{2}+\varepsilon} .
$$


As they mentioned in [10], it holds that $\zeta_{2}(i t, i t) \ll(1+|t|)^{1+\varepsilon}$ trivially. Hence (1.6) is far from the true order of magnitude for the double zeta-functions. Any other results on the order of magnitude for the double zeta-function (1.1) on the line $\sigma_{j}=\frac{1}{2}$ are not stated in [10]. In view of this, it is of some interest to try to determine an upper bound for the double zeta-function on the line other than $\sigma_{j}=0$.

In this paper, we shall study the order of magnitude of the double zeta-function (1.1) in the region $0 \leq \sigma_{j}<1(j=1,2)$, where we use, instead of Mellin-Barnes integral formula, the theory of double exponential sums of van der Corput's type (see E. Krätzel [12] and E. C. Titchmarsh [15]).

We use the standard notation e.g. $f(x)=O(g(x))$ means that $|f(x)|<C g(x)$ for $x>x_{0}$ and some constant $C>0$ where $f(x)$ is a complex function and $g(x)$ is a positive function. Further $f(x) \ll g(x)$ means the same as $f(x)=O(g(x))$ and $f(x) \asymp g(x)$ means that both $f(x) \ll g(x)$ and $g(x) \ll f(x)$ hold.

Our main result can be stated as follows.

Theorem 1.1. Let $\left|t_{1}\right|$ and $\left|t_{2}\right| \geq 2$ be real numbers such that

$$
\left|t_{1}\right| \asymp\left|t_{2}\right| \text { and }\left|t_{1}+t_{2}\right| \gg 1 \text {. }
$$

In the case $\sigma_{1}=\sigma_{2}=0$, we have

$$
\zeta_{2}\left(i t_{1}, i t_{2}\right) \ll\left|t_{1}\right| \log ^{2}\left|t_{1}\right| .
$$

Suppose that $0 \leq \sigma_{j}<1(j=1,2)$ and $\sigma_{1}+\sigma_{2}>0$. Then we have

$$
\zeta_{2}\left(\sigma_{1}+i t_{1}, \sigma_{2}+i t_{2}\right) \ll \begin{cases}\left|t_{1}\right|^{1-\frac{2}{3}\left(\sigma_{1}+\sigma_{2}\right)} \log ^{2}\left|t_{1}\right| & \left(0 \leq \sigma_{1} \leq \frac{1}{2}, 0 \leq \sigma_{2} \leq \frac{1}{2}\right) \\ \left|t_{1}\right|^{\frac{5}{6}-\frac{1}{3}\left(\sigma_{1}+2 \sigma_{2}\right)} \log ^{3}\left|t_{1}\right| & \left(\frac{1}{2}<\sigma_{1}<1,0 \leq \sigma_{2} \leq \frac{1}{2}\right) \\ \left|t_{1}\right|^{\frac{5}{6}-\frac{1}{3}\left(2 \sigma_{1}+\sigma_{2}\right)} \log ^{3}\left|t_{1}\right| & \left(0 \leq \sigma_{1} \leq \frac{1}{2}, \frac{1}{2}<\sigma_{2}<1\right) \\ \left|t_{1}\right|^{\frac{2}{3}-\frac{1}{3}\left(\sigma_{1}+\sigma_{2}\right)} \log ^{4}\left|t_{1}\right| & \left(\frac{1}{2}<\sigma_{1}<1, \frac{1}{2}<\sigma_{2}<1\right) .\end{cases}
$$

As an immediate consequence we have:

Corollary 1.2. Suppose the same condition on $t_{1}$ and $t_{2}$ as in the above theorem. Then we have,

$$
\begin{aligned}
& \zeta_{2}\left(\frac{1}{2}+i t_{1}, \frac{1}{2}+i t_{2}\right) \ll\left|t_{1}\right|^{\frac{1}{3}} \log ^{2}\left|t_{1}\right| \\
& \zeta_{2}\left(0+i t_{1}, \frac{1}{2}+i t_{2}\right) \ll\left|t_{1}\right|^{\frac{2}{3}} \log ^{2}\left|t_{1}\right| \\
& \zeta_{2}\left(\frac{1}{2}+i t_{1}, 0+i t_{2}\right) \ll\left|t_{1}\right|^{\frac{2}{3}} \log ^{2}\left|t_{1}\right| .
\end{aligned}
$$


Remark 1.3. Under the condition $\left|t_{1}\right| \asymp\left|t_{2}\right|$ and $\left|t_{1}+t_{2}\right| \gg 1$, we can expect that

$$
\zeta_{2}\left(s_{1}, s_{2}\right) \ll\left|t_{1}\right|^{\mu\left(\sigma_{1}\right)+\mu\left(\sigma_{2}\right)} \log ^{A}\left|t_{1}\right|
$$

for some constant $A$. The non-trivial estimates of the Riemann zeta-function on the imaginary axis and the critical line are $\mu(0)=\frac{1}{2}$ and $\mu\left(\frac{1}{2}\right) \leq \frac{1}{6}$, respectively. The exponents in Corollary 1.2 can be said to correspond to the classical estimates of the Riemann zeta-function.

Our theorem has an application to the modified weighted divisor problem. Let $1 \leq a \leq b$ be fixed integers, and $d(a, b ; n)$ the number of representations of $n$ as $n=n_{1}^{a} \bar{n}_{2}^{b}$, where $n_{1}$ and $n_{2}$ are positive integers. This function plays an important role in many problems. J. L. Hafner [7] and A. Ivić [11, Chapter 14] considered the asymptotic behaviour of the sum $\sum_{n \leq x} d(a, b ; n)$ whose main term can be obtained by the residue of $\zeta(a s) \zeta(b s)$ since

$$
\sum_{n=1}^{\infty} \frac{d(a, b ; n)}{n^{s}}=\zeta(a s) \zeta(b s) \quad \Re s>1 / a .
$$

The above representation reveals the close connection between the weighted divisor problem and the Riemann zeta-function.

Now let $h(a, b ; n)$ be the number of representations of $n$ as $n=n_{1}^{a} n_{2}^{b}$ with $n_{1}<n_{2}$ :

$$
h(a, b ; n)=\sum_{\substack{n=n_{1}^{a} n_{2}^{b} \\ n_{1}<n_{2}}} 1 .
$$

In this case we have

$$
\sum_{n=1}^{\infty} \frac{h(a, b ; n)}{n^{s}}=\zeta_{2}(a s, b s)
$$

for $\Re s>\max \{2 /(a+b), 1 / b\}$. Our estimate can be applied to the analysis of $\sum_{n \leq x} h(a, b ; n)$, which will be considered elsewhere.

ACKNOWLEDGEMEnTs. The authors would like to express their gratitude to Professor Andrzej Schinzel for pointing them Huxley's new result on $\mu(1 / 2)$ and to Professor Chaohua Jia for the valuable discussions. They are also grateful to the referee for valuable comments.

\section{Some lemmas on the Riemann zeta-function}

Our proof of Theorem 1.1 depends on the expression derived from the EulerMaclaurin summation formula. Usual formula, however, is not enough for our purpose, so we will give some refinement of it in the following lemma. 
We prepare some notation. Let $B_{r}$ and $B_{r}(x)$ denote the $r$-th Bernoulli number and $r$-th Bernoulli polynomial, respectively. We put $\bar{B}_{r}(x)=B_{r}(x-[x])$ as usual. Let $\Gamma(a, z)$ and $\Psi(a, b ; z)$ denote the incomplete Gamma function of the second kind and one of the solutions of confluent hypergeometric equation defined by

$$
\Gamma(a, z)=\int_{z}^{\infty} e^{-t} t^{a-1} d t, \quad \Re(a)>0,
$$

and

$$
\begin{aligned}
\Psi(a, b ; z)= & \frac{\Gamma(1-b)}{\Gamma(a-b+1)}{ }_{1} F_{1}(a, b ; z) \\
& +\frac{\Gamma(b-1)}{\Gamma(a)} z^{1-b}{ }_{1} F_{1}(a-b+1,2-b ; z),
\end{aligned}
$$

respectively. These two functions are connected by the relation

$$
\Gamma(a, z)=z^{a} e^{-z} \Psi(1, a+1 ; z)
$$

(see A. Erdélyi et al. [4, p. 257 (6) and p. 266 (21)]). The integral representation

$$
\Psi(a, c ; z)=\frac{1}{\Gamma(a)} \int_{0}^{\infty e^{i \phi}} e^{-z t} t^{a-1}(1+t)^{c-a-1} d t
$$

holds true for $\Re a>0,-\pi<\phi<\pi$ and $-\frac{1}{2} \pi<\phi+\arg z<\frac{1}{2} \pi$ ([4, p. 256 (3)]). Finally let $(w)_{p}$ be the rising factorial defined by

$$
(w)_{0}=1, \quad(w)_{p+1}=(w+p)(w)_{p}
$$

for non-negative integer $p$.

Lemma 2.1. Let $\Re \mu<1$ and $x$ be real which satisfies $|x| \geq \frac{\pi}{2}|\Im \mu|$. Then we have

$$
|\Psi(1, \mu+1 ; i x)| \leq \frac{2}{|x|-|\Im \mu|} .
$$

Proof. We may suppose that $\Im \mu>0$ without loss of generality. Let

$$
J:=\Psi(1, \mu+1, i x)=\int_{0}^{\infty e^{i \phi}} e^{-i x t}(1+t)^{\mu-1} d t
$$

for simplicity, where we used the integral representation of (2.2). 
(I) The case $x>0$.

Since $\arg (i x)=\frac{\pi}{2}$, we can take $-\pi<\phi<0$. We introduce a new variable $u$ by $t=e^{i \phi} u(u \geq 0)$, thereby we have

$$
J=e^{i \phi \mu} \int_{0}^{\infty} e^{-e^{i\left(\phi+\frac{\pi}{2}\right)} x u}\left(u+e^{-i \phi}\right)^{\mu-1} d u .
$$

Putting

$$
u+e^{-i \phi}=r e^{i \xi} \quad(r \geq 1,0<\xi \leq-\phi)
$$

and noting that $\Re \mu<1$ by the assumption of this lemma, we have

$$
|J| \leq e^{-\phi \Im \mu} \int_{0}^{\infty} e^{-x u \cos \left(\frac{\pi}{2}+\phi\right)} e^{-\xi \Im \mu} d u .
$$

Now we take $\phi=-\frac{\pi}{2}$, then we have $\cot \xi=u$ for this choice. To evaluate the integral (2.4), we divide the range of integration into two parts at 1 . For $0 \leq u \leq 1$, using the following inequality

$$
\xi=\operatorname{arccot} u \geq \frac{\pi}{2}-u,
$$

we have

$$
\int_{0}^{1} e^{-x u-\xi \Im \mu} d u \leq \int_{0}^{1} e^{-x u-\left(\frac{\pi}{2}-u\right) \Im \mu} d u \leq \frac{e^{-\frac{\pi}{2} \Im \mu}}{x-\Im \mu} .
$$

For $u \geq 1$, we have

$$
\int_{1}^{\infty} e^{-x u-\xi \Im \mu} d u \leq \int_{1}^{\infty} e^{-x u} d u=\frac{e^{-x}}{x} .
$$

Hence (2.5) and (2.6) give us

$$
|J| \leq e^{\frac{\pi}{2} \Im \mu}\left(\frac{e^{-\frac{\pi}{2} \Im \mu}}{x-\Im \mu}+\frac{e^{-x}}{x}\right) \leq \frac{2}{x-\Im \mu} .
$$

(II) The case $x<0$.

Since $\arg (i x)=-\frac{\pi}{2}$, we can take $0<\phi<\pi$ in this case. We put $u+e^{-i \phi}=$ $r e^{-i \xi}(0<\xi \leq \phi)$ and as in the previous case, we can easily see that

$$
|J| \leq \int_{0}^{\infty} e^{-|x| u \cos \left(-\frac{\pi}{2}+\phi\right)} e^{-(\phi-\xi) \Im \mu} d u \leq \frac{1}{|x| \cos \left(-\frac{\pi}{2}+\phi\right)} .
$$

Hence, taking $\phi=\frac{\pi}{2}$, we have

$$
|J| \leq \frac{1}{|x|}
$$

This completes the proof of the lemma. 
Lemma 2.2. Let $s=\sigma+i t,|t|>1$. For $N>\frac{1}{4}|t|, m \geq 1$ and $\sigma>-2 m-1$, we have

$$
\begin{aligned}
\zeta(s)= & \sum_{n \leq N} \frac{1}{n^{s}}+\frac{N^{1-s}}{s-1}-\frac{N^{-s}}{2}+\sum_{k=1}^{2 m} \frac{B_{k+1}}{(k+1) !}(s)_{k} N^{-(s+k)} \\
& +O\left(|t|^{2 m+1} N^{-\sigma-2 m-1}\right),
\end{aligned}
$$

where the implied constant does not depend on $t$.

To prove our theorem, we apply Lemma 2.2 in the case $m=1$ which we present as a corollary.

Corollary 2.3. Let $s=\sigma+i t,|t|>1$. For $N>\frac{1}{4}|t|$ and $\sigma>-3$, we have

$$
\zeta(s)=\sum_{n \leq N} \frac{1}{n^{s}}+\frac{N^{1-s}}{s-1}-\frac{N^{-s}}{2}+\frac{s}{12} N^{-s-1}+O\left(|t|^{3} N^{-\sigma-3}\right),
$$

where the implied constant does not depend on $t$.

Proof of Lemma 2.2. We start with the well-known formula for the Riemann zetafunction which is derived by the Euler-Maclaurin summation formula:

$$
\zeta(s)=\sum_{n \leq N} \frac{1}{n^{s}}+\frac{N^{1-s}}{s-1}-\frac{1}{2 N^{s}}+\sum_{k=1}^{M-1} \frac{B_{k+1}}{(k+1) !}(s)_{k} N^{-(s+k)}+R_{M, N},
$$

where $N$ and $M$ are positive integers and

$$
R_{M, N}=-\frac{(s)_{M}}{M !} \int_{N}^{\infty} \bar{B}_{M}(x) x^{-s-M} d x .
$$

We take $M=2 m+1$. The function $\bar{B}_{2 m+1}(x)$ is a periodic function with period 1 whose Fourier expansion is given by

$$
\bar{B}_{2 m+1}(x)=2(2 m+1) !(-1)^{m-1} \sum_{\nu=1}^{\infty} \frac{\sin 2 \pi v x}{(2 \pi v)^{2 m+1}} .
$$

Substituting (2.13) into (2.12), we have

$$
R_{2 m+1, N}=2(-1)^{m}(s)_{2 m+1} \sum_{\nu=1}^{\infty}(2 \pi \nu)^{s-1} \int_{2 \pi \nu N}^{\infty} x^{-s-2 m-1} \sin x d x .
$$

Now the last integral of (2.14) can be written as

$$
\int_{2 \pi \nu N}^{\infty} x^{\mu-1} \sin x d x=\frac{i}{2}\left\{e^{-\frac{\pi i \mu}{2}} \Gamma(\mu, 2 \pi i v N)-e^{\frac{\pi i \mu}{2}} \Gamma(\mu,-2 \pi i \nu N)\right\}
$$


for $\Re \mu<1$ (I. S. Gradshteyn and I. M. Ryzhik [5, 3.761-2]), hence putting $\mu=$ $-s-2 m$ and using Lemma 2.1, we have

$$
\int_{2 \pi v N}^{\infty} x^{-s-2 m-1} \sin x d x \ll \frac{(v N)^{-\sigma-2 m}}{2 \pi v N-|t|}
$$

for $N \geq \frac{1}{4}|t|$. Therefore we get

$$
\begin{aligned}
R_{2 m+1, N} & \ll\left|(s)_{2 m+1}\right| N^{-\sigma-2 m} \sum_{\nu=1}^{\infty} \frac{1}{v^{2 m+1}} \frac{1}{2 \pi \nu N-|t|} \\
& \ll \frac{|t|^{2 m+1}}{N^{\sigma+2 m+1}}
\end{aligned}
$$

for $N \geq \frac{1}{4}|t|$. This completes the proof of Lemma 2.2.

Remark 2.4. (i) If we evaluate the integral (2.12) directly, we only get

$$
R_{2 m+1, N} \ll \frac{|t|^{2 m+1}}{N^{\sigma+2 m}},
$$

which is not sufficient for our purpose.

(ii) The approximate functional equation in the simplest form can be written as

$$
\zeta(s)=\sum_{n \leq x} \frac{1}{n^{s}}+\frac{x^{1-s}}{s-1}+O\left(x^{-\sigma}\right)
$$

for $0<\sigma_{0} \leq \sigma \leq 2, x \geq|t| / \pi$ (A. Ivić [11, Theorem 1.8]). Lemma 2 can be regarded as a refinement of this formula.

For the estimate of finite zeta sum and the zeta-function, we shall use the following lemma.

Lemma 2.5. Let $t>2, N \leq N_{1} \leq 2 N$ and $N \ll t$, then we have

$$
\begin{gathered}
\sum_{N<n \leq N_{1}} \frac{1}{n^{1 / 2+i t}} \ll t^{\frac{1}{6}}, \\
\sum_{N<n \leq N_{1}} \frac{1}{n^{i t}} \ll t^{\frac{1}{2}}, \\
\sum_{N<n \leq N_{1}} \frac{1}{n^{s}} \ll \begin{cases}t^{\frac{1}{2}-\frac{2}{3} \sigma} & \left(0<\sigma<\frac{1}{2}\right) \\
t^{\frac{1}{3}-\frac{1}{3} \sigma} \log t & \left(\frac{1}{2}<\sigma \leq 1\right),\end{cases}
\end{gathered}
$$


and

$$
\zeta(\sigma+i t) \ll \begin{cases}t^{\frac{1}{2}-\frac{2}{3} \sigma} \log t & \left(0 \leq \sigma \leq \frac{1}{2}\right) \\ t^{\frac{1}{3}-\frac{1}{3} \sigma} \log ^{2} t & \left(\frac{1}{2}<\sigma \leq 1\right) .\end{cases}
$$

Proof. For the proof of (2.18) and (2.19), see E. C. Titchmarsh [16, Theorem 5.12] and S. W. Graham and G. Kolesnik [6, Theorem 2.2]. As for (2.20), we use (2.18), (2.19), trivial estimate $\sum_{n \leq N} \frac{1}{n^{1+i t}} \ll \log N$ and the Phragmén-Lindelöf convexity principal.

From Corollary 2.3, we get

$$
\zeta(s)=\sum_{n \leq N} \frac{1}{n^{s}}+O\left(t^{-\sigma}\right) \quad \text { for } \quad \frac{t}{4}<N \ll t .
$$

Hence by dividing the range $1 \leq n \leq N$ into $O(\log t)$ subsums of the form of the left-hand side of the above, we obtain (2.21).

\section{Double exponential sums}

Next we shall recall the simplest result for double exponential sum, which is given by E. Krätzel [12, p. 61] and E. C. Titchmarsh [15]. Throughout this paper the following conditions are always assumed to be true:

(A) Suppose that $D$ is a subset of the rectangle

$$
D_{1}=\left\{\left(x_{1}, x_{2}\right) \mid a_{1} \leq x_{1} \leq b_{1}, a_{2} \leq x_{2} \leq b_{2}\right\}
$$

with $c_{j}:=b_{j}-a_{j} \geq 1(j=1,2)$, where $D$ denotes a bounded plane domain with the area $|D|$.

(B) Any straight line parallel to any of coordinate axis intersects $D$ in a bounded number of line segment. For the sake of simplicity we only consider such domains $D$ where these straight lines intersects the boundary of $D$ in at most two points or in one line segments. We can do this without loss of generality, because each such general domain can be divided into a finite number of these special domains.

(C) Let $f\left(x_{1}, x_{2}\right)$ be a real function in $D_{1}$ with continuous partial derivatives of as many orders as may be required. Suppose that the functions $f_{x_{1}}\left(x_{1}, x_{2}\right)$ and $f_{x_{2}}\left(x_{1}, x_{2}\right)$ are monotonic in $x_{1}$ and $x_{2}$, respectively.

(D) Intersections of $D$ with domains of the type $f_{x_{j}}\left(x_{1}, x_{2}\right) \leq c$ or $f_{x_{2}}\left(x_{1}, x_{2}\right) \leq$ $c(j=1,2)$ must satisfy condition (B) as well.

(E) The boundary of $D$ can be divided into a bounded number of parts. In each part the curve of boundary is given by $x_{2}=$ constant or a function $x_{1}=\rho\left(x_{2}\right)$, which is continuous in the closed intervals described above.

We need the following lemmas. 
Lemma 3.1 (Titchmarsh [15, Lemma $\gamma]$ ). Let $f\left(x_{1}, x_{2}\right)$ be a real differentiable function of $x_{1}$ and $x_{2}$. Let $f_{x_{1}}\left(x_{1}, x_{2}\right)$, as a function of $x_{1}$ for each fixed value of $x_{2}$, have a finite number of maxima and minima, and let $f_{x_{2}}\left(x_{1}, x_{2}\right)$ satisfy a similar condition as a function of $x_{2}$ for each fixed value of $x_{1}$. Let $0<\delta<1$ be a fixed number and let

$$
\left|f_{x_{1}}\left(x_{1}, x_{2}\right)\right| \leq \delta, \quad\left|f_{x_{2}}\left(x_{1}, x_{2}\right)\right| \leq \delta
$$

for $\left(x_{1}, x_{2}\right) \in D$. Then

$$
\sum_{\left(n_{1}, n_{2}\right) \in D} e^{2 \pi i f\left(n_{1}, n_{2}\right)}=\iint_{D} e^{2 \pi i f\left(x_{1}, x_{2}\right)} d x_{1} d x_{2}+O\left(c_{1}\right)+O\left(c_{2}\right) .
$$

Lemma 3.2 (Krätzel [12, Theorem 2.21]). Let $f\left(x_{1}, x_{2}\right)$ be a real function in $D^{\prime}$, and let $H_{1}, H_{2}$ be integers with $1 \leq H_{j} \leq c_{j}(j=1,2)$. Let

$$
W=\sum_{\left(n_{1}, n_{2}\right) \in D} e^{2 \pi i f\left(n_{1}, n_{2}\right)}
$$

Then we have

$$
W \ll \frac{\left|D^{\prime}\right|}{\sqrt{H_{1} H_{2}}}+\left\{\frac{\left|D^{\prime}\right|}{H_{1} H_{2}} \sum_{h_{1}=1}^{H_{1}-1} \sum_{h_{2}=0}^{H_{2}-1}\left|W_{1}\right|\right\}^{1 / 2}+\left\{\frac{\left|D^{\prime}\right|}{H_{1} H_{2}} \sum_{h_{1}=0}^{H_{1}-1} \sum_{h_{2}=1}^{H_{2}-1}\left|W_{2}\right|\right\}^{1 / 2},
$$

where

$$
W_{1}=\sum_{\substack{\left(n_{1}, n_{2}\right) \in D \\\left(n_{1}+h_{1}, n_{2}+h_{2}\right) \in D}} e^{2 \pi i\left(f\left(n_{1}+h_{1}, n_{2}+h_{2}\right)-f\left(n_{1}, n_{2}\right)\right)}
$$

and

$$
W_{2}=\sum_{\substack{\left(n_{1}, n_{2}\right) \in D \\\left(n_{1}+h_{1}, n_{2}-h_{2}\right) \in D}} e^{2 \pi i\left(f\left(n_{1}+h_{1}, n_{2}-h_{2}\right)-f\left(n_{1}, n_{2}\right)\right)} .
$$

Further, we denote the Hessian of the function $f\left(x_{1}, x_{2}\right)$ by

$$
H(f)=\frac{\partial\left(f_{x_{1}}, f_{x_{2}}\right)}{\partial\left(x_{1}, x_{2}\right)}=f_{x_{1} x_{1}}\left(x_{1}, x_{2}\right) f_{x_{2} x_{2}}\left(x_{1}, x_{2}\right)-f_{x_{1} x_{2}}^{2}\left(x_{1}, x_{2}\right) .
$$

Lemma 3.3 (Krätzel [12, Lemma 2.6]). Suppose that

$$
\lambda_{j} \leq\left|f_{x_{j} x_{j}}\left(x_{1}, x_{2}\right)\right| \ll \lambda_{j}(j=1,2), \quad\left|f_{x_{1} x_{2}}\left(x_{1}, x_{2}\right)\right| \ll \sqrt{\lambda_{1} \lambda_{2}}
$$

and

$$
H(f) \gg \lambda_{1} \lambda_{2}
$$


throughout the rectangle $D_{1}$. For all parts of the curve of boundary let $x_{2}=\mathrm{const}$ or $x_{1}=\rho\left(x_{2}\right)$, where $\rho(x)$ is partly twice differential and $\left|\rho^{\prime \prime}(x)\right| \ll r$. Then we have

$$
\iint_{D} e^{2 \pi i f\left(x_{1}, x_{2}\right)} d x_{1} d x_{2} \ll \frac{1+\log \left|D_{1}\right|+\left|\log \lambda_{1}\right|+\left|\log \lambda_{2}\right|}{\sqrt{\lambda_{1} \lambda_{2}}}+\frac{c_{2} r}{\lambda_{2}} .
$$

Lemma 3.4 (Krätzel [12, Theorem 2.16]). Suppose that

$$
\left|f_{x_{j} x_{j}}\left(x_{1}, x_{2}\right)\right| \asymp \lambda_{j}(j=1,2), \quad\left|f_{x_{1} x_{2}}\left(x_{1}, x_{2}\right)\right| \ll \sqrt{\lambda_{1} \lambda_{2}}
$$

and

$$
|H(f)| \gg \lambda_{1} \lambda_{2}
$$

throughout the rectangle $D_{1}$. For all parts of the curve of boundary let $x_{2}=$ const or $x_{1}=\rho\left(x_{2}\right)$, where $\rho(x)$ is partly twice differentiable and $\left|\rho^{\prime \prime}(x)\right| \ll r$. If $R$ is defined by

$$
R=1+\log \left|D_{1}\right|+\left|\log \lambda_{1}\right|+\left|\log \lambda_{2}\right|+c_{2} r \sqrt{\frac{\lambda_{1}}{\lambda_{2}}}
$$

then we have

$$
\begin{aligned}
& \sum_{\left(n_{1}, n_{2}\right) \in D} e\left(f\left(n_{1}, n_{2}\right)\right) \\
& \ll\left(c_{1} \lambda_{1}+c_{2} \sqrt{\lambda_{1} \lambda_{2}}+1\right)\left(c_{2} \lambda_{2}+c_{1} \sqrt{\lambda_{1} \lambda_{2}}+1\right) \frac{R}{\sqrt{\lambda_{1} \lambda_{2}}} .
\end{aligned}
$$

Let $M$ and $N$ be positive integers such that $M<N$. In the next lemma we shall give the partial summation formula for the double sum $\sum_{M<m \leq n \leq N} f(m, n) g(m, n)$ where $f(x, y)$ is a $C^{2}$-function on $[M, N] \times[M, N]$ and $g(m, n)$ is an arithmetical function on the same domain. Let

$$
G(x, y)=\sum_{x<m \leq n \leq y} g(m, n)
$$

Lemma 3.5. Let the notation be as above. Suppose that

$$
\begin{array}{ll}
|G(x, y)| \leq G, & \left|f_{x}(x, y)\right| \leq \kappa_{1} \\
\left|f_{y}(x, y)\right| \leq \kappa_{2}, & \left|f_{x y}(x, y)\right| \leq \kappa_{3}
\end{array}
$$

for any $M \leq x, y \leq N$. 
Then we have

$$
\begin{aligned}
& \left|\sum_{M<m \leq n \leq N} f(m, n) g(m, n)\right| \\
& \quad \leq G\left(|f(M, N)|+\left(\kappa_{1}+\kappa_{2}\right)(N-M)+\kappa_{3}(N-M)^{2}\right) .
\end{aligned}
$$

Proof. We shall apply partial summation twice. Let

$$
V(n)=\sum_{M<m \leq n} f(m, n) g(m, n) .
$$

Then we can write

$$
J:=\sum_{M<m \leq n \leq N} f(m, n) g(m, n)=\sum_{M<n \leq N} V(n) .
$$

By using partial summation to the sum $V(n)$, we have

$$
V(n)=f(n, n) H(n, n)-\int_{M}^{n} f_{x}(x, n) H(x, n) d x
$$

with

$$
H(x, n)=\sum_{M<m \leq x} g(m, n) .
$$

Substituting (3.5) into (3.4), we have

$$
\begin{aligned}
J & =\sum_{M<n \leq N} f(n, n) H(n, n)-\sum_{M<n \leq N} \int_{M}^{n} f_{x}(x, n) H(x, n) d x \\
& =J_{1}-J_{2},
\end{aligned}
$$

say. We apply partial summation again in the sums of $J_{1}$ and $J_{2}$, namely we have

$$
\begin{aligned}
J_{1} & =f(N, N) \sum_{M<n \leq N} H(n, n)-\int_{M}^{N} \frac{d}{d x} f(x, x)\left(\sum_{M<n \leq x} H(n, n)\right) d x \\
& =f(N, N) G(M, N)-\int_{M}^{N} \frac{d}{d x} f(x, x) G(M, x) d x \\
& =f(N, N) G(M, N)-\int_{M}^{N}\left(f_{x}(x, x)+f_{y}(x, x)\right) G(M, x) d x,
\end{aligned}
$$


and

$$
\begin{aligned}
J_{2}= & \int_{M}^{N} \sum_{x<n \leq N} f_{x}(x, n) H(x, n) d x \\
= & \int_{M}^{N}\left\{f_{x}(x, N) \sum_{x<n \leq N} H(x, n)-\int_{x}^{N} f_{x y}(x, y)\left(\sum_{x<n \leq y} H(x, n)\right) d y\right\} d x \\
= & \int_{M}^{N}\left\{f_{x}(x, N)(G(M, N)-G(M, x)-G(x, N))\right. \\
& \left.-\int_{x}^{N} f_{x y}(x, y)(G(M, y)-G(M, x)-G(x, y)) d y\right\} d x \\
= & G(M, N)(f(N, N)-f(M, N)) \\
& -\int_{M}^{N}\left(f_{x}(x, N) G(x, N)+f_{x}(x, x) G(M, x)\right) d x \\
& -\int_{M}^{N} \int_{x}^{N} f_{x y}(x, y)(G(M, y)-G(x, y)) d y d x .
\end{aligned}
$$

Hence we have

$$
\begin{aligned}
J= & f(M, N) G(M, N)+\int_{M}^{N}\left(f_{x}(x, N) G(x, N)-f_{y}(x, x) G(M, x)\right) d x \\
& +\int_{M}^{N} \int_{x}^{N} f_{x y}(x, y)(G(M, y)-G(x, y)) d y d x .
\end{aligned}
$$

Our assertion follows by taking the absolute value in the right-hand side of (3.6).

\section{Proof of Theorem 1.1}

Let $s_{j}=\sigma_{j}+i t_{j}(j=1,2)$ be complex variables with $\left|t_{1}\right| \asymp\left|t_{2}\right|$. We take a parameter $\tau$ such that $\max \left\{\left|t_{1}\right|,\left|t_{2}\right|,\left|t_{1}+t_{2}\right|\right\}+2 \leq \tau \ll\left|t_{1}\right|$.

Assuming that $\Re s_{j}=\sigma_{j}>1 \quad(j=1,2)$, we divide the double series (1.1) as

$$
\begin{aligned}
\zeta_{2}\left(s_{1}, s_{2}\right) & =\sum_{m<n \leq \tau} \frac{1}{m^{s_{1}} n^{s_{2}}}+\sum_{\substack{m<n \\
n>\tau}} \frac{1}{m^{s_{1}} n^{s_{2}}} \\
& =: S_{1}\left(s_{1}, s_{2}\right)+S_{2}\left(s_{1}, s_{2}\right),
\end{aligned}
$$

say. After analytic continuation of the infinite sum $S_{2}\left(s_{1}, s_{2}\right)$, we consider the order of magnitude of these sums in the range

$$
0 \leq \sigma_{j}<1(j=1,2) .
$$




\subsection{Evaluation of $S_{2}\left(s_{1}, s_{2}\right)$}

First we shall consider the estimate of $S_{2}\left(s_{1}, s_{2}\right)$. Since $n$ runs over the integers greater than $\tau>\left|t_{1}\right|$, we can use Corollary 2.3 to obtain

$$
\begin{aligned}
S_{2}\left(s_{1}, s_{2}\right)= & \sum_{n>\tau} \frac{1}{n^{s_{2}}}\left(\sum_{m \leq n} \frac{1}{m^{s_{1}}}-\frac{1}{n^{s_{1}}}\right) \\
= & \zeta\left(s_{1}\right) \sum_{n>\tau} \frac{1}{n^{s_{2}}}+\frac{1}{1-s_{1}} \sum_{n>\tau} \frac{1}{n^{s_{1}+s_{2}-1}}-\frac{1}{2} \sum_{n>\tau} \frac{1}{n^{s_{1}+s_{2}}} \\
& -\frac{s_{1}}{12} \sum_{n>\tau} \frac{1}{n^{s_{1}+s_{2}+1}}+O\left(\left|s_{1}\right|^{3} \sum_{n>\tau} \frac{1}{n^{\sigma_{1}+\sigma_{2}+3}}\right) \\
= & : I_{1}+I_{2}+I_{3}+I_{4}+I_{5},
\end{aligned}
$$

say.

Since $\sigma_{1}+\sigma_{2} \geq 0$, the sum in $I_{5}$ converges absolutely, and we have

$$
I_{5} \ll \tau^{1-\left(\sigma_{1}+\sigma_{2}\right)} .
$$

The analytic continuation and the estimate of the sum $\sum_{n>\tau} \frac{1}{n^{w}}$ in the range $\Re w \leq$ 1 are also given by Corollary 2.3 under the condition $\tau>|\Im w w| / 4$. For the estimate of $I_{1}$, we have

$$
\begin{aligned}
I_{1} & =\zeta\left(s_{1}\right)\left\{\frac{\tau^{1-s_{2}}}{s_{2}-1}-\frac{1}{2} \tau^{-s_{2}}+\frac{s_{2}}{12} \tau^{-s_{2}-1}+O\left(\tau^{-\sigma_{2}}\right)\right\} \\
& \ll\left|\zeta\left(s_{1}\right)\right| \tau^{-\sigma_{2}},
\end{aligned}
$$

and for $I_{2}$, we have

$$
\begin{aligned}
I_{2}= & \frac{1}{1-s_{1}}\left\{\frac{\tau^{2-\left(s_{1}+s_{2}\right)}}{s_{1}+s_{2}-2}-\frac{1}{2} \tau^{1-\left(s_{1}+s_{2}\right)}\right. \\
& \left.\quad+\frac{s_{1}+s_{2}-1}{12} \tau^{-\left(s_{1}+s_{2}\right)}+O\left(\tau^{1-\left(\sigma_{1}+\sigma_{2}\right)}\right)\right\} \\
& \ll \tau^{1-\left(\sigma_{1}+\sigma_{2}\right)} .
\end{aligned}
$$

Similarly we have

$$
I_{j} \ll \tau^{1-\left(\sigma_{1}+\sigma_{2}\right)}, \quad(j=3,4) .
$$

Combining (4.3), (4.4), (4.5) and (4.6), we have

$$
S_{2}\left(s_{1}, s_{2}\right) \ll \tau^{\max \left\{\mu\left(\sigma_{1}\right), 1-\sigma_{1}\right\}-\sigma_{2}},
$$

in particular, for $\sigma_{1}<1$,

$$
S_{2}\left(s_{1}, s_{2}\right) \ll \tau^{1-\left(\sigma_{1}+\sigma_{2}\right)} .
$$




\subsection{Evaluation of $S_{1}\left(s_{1}, s_{2}\right)$}

We shall consider the estimate of $S_{1}\left(s_{1}, s_{2}\right)$. Let $2 \leq M \leq \tau / 2$. We define, for $\sigma_{j} \geq 0(j=1,2)$,

$$
T\left(s_{1}, s_{2} ; M\right)=\sum_{M<m<n \leq 2 M} \frac{1}{m^{s_{1}} n^{s_{2}}}
$$

and

$$
U\left(s_{1}, s_{2} ; M\right)=\sum_{m \leq M} \frac{1}{m^{s_{1}}} \sum_{M<n \leq 2 M} \frac{1}{n^{s_{2}}} .
$$

Since $S_{1}$ can be written as

$$
S_{1}\left(s_{1}, s_{2}\right)=\sum_{j=1}^{\left[\frac{\log 2 \tau}{\log 2}\right]}\left\{T\left(s_{1}, s_{2} ; 2^{-j} \tau\right)+U\left(s_{1}, s_{2} ; 2^{-j} \tau\right)\right\},
$$

it is enough to consider the estimates for $T\left(s_{1}, s_{2} ; M\right)$ and $U\left(s_{1}, s_{2} ; M\right)$.

First we consider the case $\sigma_{1}=\sigma_{2}=0$. Applying Lemma 3.4 to the function $f\left(x_{1}, x_{2}\right)=-\frac{1}{2 \pi}\left(t_{1} \log x_{1}+t_{2} \log x_{2}\right)$ and noting that $\tau \asymp\left|t_{j}\right|$ and $M \leq \tau / 2$, we have

$$
T\left(i_{1}, i t_{2} ; M\right) \ll \tau \log \tau .
$$

As for the term $U\left(i t_{1}, i t_{2} ; M\right)$, we have from (2.19)

$$
U\left(i t_{1}, i t_{2} ; M\right) \ll \tau \log \tau .
$$

From (4.8), (4.9) and (4.10), we have

$$
S_{1}\left(i t_{1}, i t_{2}\right) \ll \tau \log ^{2} \tau .
$$

The proof of (1.7) follows from (4.11) in conjunction with (4.7).

Next we consider the case of $\sigma_{1}+\sigma_{2}>0$.

Estimation of $T\left(s_{1}, s_{2} ; M\right)$. To consider the upper bounds for $T\left(s_{1}, s_{2} ; M\right)$, we divide the region into three parts:

$$
M \ll \tau^{\frac{1}{3}}, \tau^{\frac{1}{3}} \ll M \ll \tau^{\frac{2}{3}}, \tau^{\frac{2}{3}} \ll M \ll \tau .
$$

Let $j_{0}=[\log \tau / 3 \log 2]$ and $N=2^{-j_{0}} \tau \asymp \tau^{2 / 3}$. To reduce the evaluation of $T\left(\sigma_{1}+i t_{1}, \sigma_{2}+i t_{2} ; M\right)$ into that of $T\left(i t_{1}, i t_{2} ; M\right)$, we apply Lemma 3.5 with $f(x, y)=\frac{1}{x^{\sigma_{1}} y^{\sigma_{2}}}$ and $g(m, n)=e^{-i\left(t_{1} \log m+t_{2} \log n\right)}$. Thus we have

$$
\begin{aligned}
& T\left(s_{1}, s_{2} ; M\right) \\
& =\sum_{M<m \leq n \leq 2 M} \frac{1}{m^{\sigma_{1}+i t_{1}} n^{\sigma_{2}+i t_{2}}}-\sum_{M<n \leq 2 M} \frac{1}{n^{\sigma_{1}+\sigma_{2}+i\left(t_{1}+t_{2}\right)}} \\
& \ll M^{-\sigma_{1}-\sigma_{2}}\left\{\max _{M<x<y \leq 2 M}\left|\sum_{x<m \leq n \leq y} \frac{1}{m^{i t_{1}} n^{i t_{2}}}\right|+\max _{M<u \leq 2 M}\left|\sum_{M<n \leq u} \frac{1}{n^{i\left(t_{1}+t_{2}\right)}}\right|\right\},
\end{aligned}
$$


and hence

$$
\sum_{j \leq j_{0}} T\left(s_{1}, s_{2} ; 2^{-j} \tau\right) \ll \frac{1}{N^{\sigma_{1}+\sigma_{2}}} \tau \log ^{2} \tau \ll \tau^{1-\frac{2}{3}\left(\sigma_{1}+\sigma_{2}\right)} \log ^{2} \tau
$$

by (2.19) and (4.9).

On the other hand, for $M \ll \tau^{1 / 3}$, it follows that

$$
T\left(s_{1}, s_{2} ; M\right)=\sum_{M<m<n \leq 2 M} \sum_{m^{s_{1}} n^{s_{2}}} \ll M^{2-\sigma_{1}-\sigma_{2}} \log M .
$$

We take $j_{1}=[2 \log \tau / 3 \log 2]$, then

$$
\sum_{j>j_{1}} T\left(s_{1}, s_{2} ; 2^{-j} \tau\right) \ll \tau^{\frac{2}{3}-\frac{1}{3}\left(\sigma_{1}+\sigma_{2}\right)} \log \tau
$$

We use Lemma 3.2 with $H_{1}=H_{2}=H$ to consider the estimate of $T\left(s_{1}, s_{2} ; M\right)$ for $\tau^{1 / 3} \ll M \ll \tau^{2 / 3}$, where $H$ is chosen later. Let $M<M^{\prime} \leq 2 M$ and

$$
W=\sum_{M<m<n \leq M^{\prime}} m^{i t_{1}} n^{i t_{2}}=\sum_{M<m<n \leq M^{\prime}} e^{2 \pi i f(m, n)},
$$

where we put

$$
f\left(x_{1}, x_{2}\right)=\frac{1}{2 \pi}\left(t_{1} \log x_{1}+t_{2} \log x_{2}\right) .
$$

For each $1 \leq h_{j} \leq H(j=1,2)$, we define

$$
D_{h_{1}, h_{2}}=\left\{(m, n) \in \mathbb{Z}^{2} \mid M<m<n \leq M^{\prime}, M<m+h_{1}<n+h_{2}<M^{\prime}\right\}
$$

and

$$
D_{h_{1}, h_{2}}^{\prime}=\left\{(m, n) \in \mathbb{Z}^{2} \mid M<m<n \leq M^{\prime}, M<m+h_{1}<n-h_{2}<M^{\prime}\right\} .
$$

By Lemma 3.2, we have

$$
W \ll \frac{M^{2}}{H}+\frac{M}{H}\left\{\left(\sum_{h_{1}=1}^{H-1} \sum_{h_{2}=0}^{H-1}\left|W_{1}\left(h_{1}, h_{2}\right)\right|\right)^{\frac{1}{2}}+\left(\sum_{h_{1}=0}^{H-1} \sum_{h_{2}=1}^{H-1}\left|W_{2}\left(h_{1}, h_{2}\right)\right|\right)^{\frac{1}{2}}\right\},
$$

where

$$
W_{1}\left(h_{1}, h_{2}\right)=\sum_{(m, n) \in D_{h_{1}, h_{2}}} e^{2 \pi i\left(f\left(m+h_{1}, n+h_{2}\right)-f(m, n)\right)}
$$

and

$$
W_{2}\left(h_{1}, h_{2}\right)=\sum_{(m, n) \in D_{h_{1}, h_{2}}^{\prime}} e^{2 \pi i\left(f\left(m+h_{1}, n-h_{2}\right)-f(m, n)\right)} .
$$


Now we treat the sum $W_{1}$. Denote

$$
g\left(x_{1}, x_{2}\right)=f\left(x_{1}+h_{1}, x_{2}+h_{2}\right)-f\left(x_{1}, x_{2}\right),
$$

then

$$
\begin{gathered}
g_{x_{1}}\left(x_{1}, x_{2}\right)=\frac{t_{1}}{2 \pi}\left(\frac{1}{x_{1}+h_{1}}-\frac{1}{x_{1}}\right), g_{x_{2}}\left(x_{1}, x_{2}\right)=\frac{t_{2}}{2 \pi}\left(\frac{1}{x_{2}+h_{2}}-\frac{1}{x_{2}}\right) \\
g_{x_{1} x_{1}}\left(x_{1}, x_{2}\right)=\frac{t_{1}}{2 \pi} \frac{h_{1}\left(2 x_{1}+h_{1}\right)}{x_{1}^{2}\left(x_{1}+h_{1}\right)^{2}}, \quad g_{x_{2} x_{2}}\left(x_{1}, x_{2}\right)=\frac{t_{2}}{2 \pi} \frac{h_{2}\left(2 x_{2}+h_{2}\right)}{x_{2}^{2}\left(x_{2}+h_{2}\right)^{2}} .
\end{gathered}
$$

Consider the case $h_{2} \neq 0$ firstly. We divide the triangular region $D_{h_{1}, h_{2}}$ into the squares of side $l$, or parts of such squares

$$
\Delta_{p, q}=\{(x, y) \mid M+p l<x \leq M+(p+1) l, M+q l<y \leq M+(q+1) l\} \cap D_{h_{1}, h_{2}}
$$

with

$$
l=\frac{A M^{3}}{\tau H}
$$

where $A$ is a small constant.

For a fixed $(\alpha, \beta) \in \Delta_{p, q}$, we get

$$
g_{x_{1}}\left(x_{1}, x_{2}\right)-g_{x_{1}}(\alpha, \beta) \ll \frac{\tau H}{2 \pi} \frac{l M}{M^{2}}=A,
$$

and if $A$ is small enough, the total variation of $g_{x_{1}}$ in $\Delta_{p, q}$ is smaller than $\frac{3}{4}$, and so is $g_{x_{2}}$. Hence there are two integers $P$ and $Q$ such that

$$
\left|g_{x_{1}}\left(x_{1}, x_{2}\right)-P\right| \leq \frac{3}{4} \text { and }\left|g_{x_{2}}\left(x_{1}, x_{2}\right)-Q\right| \leq \frac{3}{4}
$$

for any $\left(x_{1}, x_{2}\right) \in \Delta_{p, q}$. Now putting

$$
G\left(x_{1}, x_{2}\right)=g\left(x_{1}, x_{2}\right)-2 \pi\left(P x_{1}+Q x_{2}\right),
$$

then we have, from Lemma 3.1,

$$
\sum_{(m, n) \in \Delta_{p, q}} e^{2 \pi i g(m, n)}=\iint_{\Delta_{p, q}} e^{2 \pi i G\left(x_{1}, x_{2}\right)} d x_{1} d x_{2}+O(l) .
$$

Since

$$
G_{x_{1} x_{1}}\left(x_{1}, x_{2}\right) \asymp \lambda_{j}=\frac{\tau h_{j}}{M^{3}} \quad(j=1,2),
$$

by applying Lemma 3.3, we have

$$
\iint_{\Delta_{p, q}} e^{2 \pi i G(x, y)} d x d y \ll \frac{\log \tau}{\tau} \frac{M^{3}}{\sqrt{h_{1} h_{2}}} .
$$


Now the number of $\Delta_{p, q}$ is at most $O\left(M^{2} / l^{2}\right)$, thus we have

$$
\begin{aligned}
W_{1}\left(h_{1}, h_{2}\right) & \ll \sum_{p, q}\left|\iint_{\Delta_{p, q}} e^{2 \pi i G(x, y)} d x d y+O(l)\right| \\
& \ll\left(\frac{\log \tau}{\tau} \frac{M^{3}}{\sqrt{h_{1} h_{2}}}+l\right) \frac{M^{2}}{l^{2}} \\
& \ll \tau \log \tau \frac{H^{2}}{\sqrt{h_{1} h_{2}}} \frac{1}{M} .
\end{aligned}
$$

by (4.15).

When $h_{2}=0$, we have, by E. Krätzel [12, Theorem 2.1],

$$
\begin{aligned}
W_{1}\left(h_{1}, 0\right) & =\sum_{M+h_{1}<n \leq M^{\prime}} \sum_{M<m<n} e^{i t_{1}\left(\log \left(m+h_{1}\right)-\log m\right)} \\
& \ll \sum_{M+h_{1}<n \leq M^{\prime}} \frac{\tau h_{1} / M^{2}}{\sqrt{\tau h_{1} / M^{3}}} \\
& \ll \sqrt{\tau M h_{1}}
\end{aligned}
$$

Similar estimates can be obtained for $W_{2}\left(h_{1}, h_{2}\right)$.

Therefore, we obtain

$$
\begin{aligned}
W \ll \frac{M^{2}}{H}+\frac{M}{H}\left\{\left(\sum_{h_{1}=1}^{H-1} \sum_{h_{=}}^{H-1} \frac{\tau \log \tau}{M} \frac{H^{2}}{\sqrt{h_{1} h_{2}}}\right)^{\frac{1}{2}}\right. \\
\left.+\left(\sum_{h_{1}=1}^{H-1} \sqrt{\tau M h_{1}}\right)^{\frac{1}{2}}+\left(\sum_{h_{2}=1}^{H-1} \sqrt{\tau M h_{2}}\right)^{\frac{1}{2}}\right\} \\
\ll \frac{M^{2}}{H}+(M H \tau \log \tau)^{\frac{1}{2}}+\left(\frac{\tau M^{5}}{H}\right)^{\frac{1}{4}} .
\end{aligned}
$$

Taking $H=M / \tau^{1 / 3}$, we get

$$
W \ll M \tau^{\frac{1}{3}}(\log \tau)^{\frac{1}{2}} .
$$

By Lemma 3.5 and (4.16), we have

$$
T\left(s_{1}, s_{2} ; M\right) \ll M^{1-\sigma_{1}-\sigma_{2}} \tau^{\frac{1}{3}} \log ^{\frac{1}{2}} \tau .
$$

It follows that

$$
\sum_{j_{0}<j \leq j_{1}} T\left(s_{1}, s_{2} ; 2^{-j} \tau\right) \ll \begin{cases}\tau^{1-\frac{2}{3}\left(\sigma_{1}+\sigma_{2}\right)} \log ^{\frac{3}{2}} \tau & \sigma_{1}+\sigma_{2} \leq 1 \\ \tau^{\frac{2}{3}-\frac{1}{3}\left(\sigma_{1}+\sigma_{2}\right)} \log ^{\frac{3}{2}} \tau & \sigma_{1}+\sigma_{2}>1\end{cases}
$$


From (4.12), (4.13) and (4.17) we have

$$
\sum_{j} T\left(s_{1}, s_{2} ; 2^{-j} \tau\right) \ll\left(\tau^{1-\frac{2}{3}\left(\sigma_{1}+\sigma_{2}\right)}+\tau^{\frac{2}{3}-\frac{1}{3}\left(\sigma_{1}+\sigma_{2}\right)}\right) \log ^{2} \tau .
$$

Remark 4.1. In the case $W_{1}\left(h_{1}, 0\right)$, we note that $g_{x_{2} x_{2}}=0, H(g)=0$, since $g\left(x_{1}, x_{2}\right)=\frac{t_{1}}{2 \pi}\left(\log \left(x_{1}+h_{1}\right)-\log x_{1}\right)$. This is the reason that we used E. Krätzel $\left[12\right.$, Theorem 2.1]. The situation is the same for $W_{2}\left(0, h_{2}\right)$.

Estimation of $U\left(s_{1}, s_{2} ; M\right)$

To treat the sum of $U\left(s_{1}, s_{2}, M\right)$, we shall apply Lemma 2.5. Noting that $\tau \asymp\left|t_{j}\right|$, we have

$$
\sum_{1 \leq m \leq M} \frac{1}{m^{s_{1}}} \ll \begin{cases}\tau^{\frac{1}{2}-\frac{2}{3} \sigma_{1}} \log \tau & \left(0 \leq \sigma_{1} \leq \frac{1}{2}\right) \\ \tau^{\frac{1}{3}-\frac{1}{3} \sigma_{1}} \log ^{2} \tau & \left(\frac{1}{2}<\sigma_{1}<1\right)\end{cases}
$$

and

$$
\sum_{M<n \leq 2 M} \frac{1}{n^{S_{2}}} \ll \begin{cases}\tau^{\frac{1}{2}-\frac{2}{3} \sigma_{2}} & \left(0 \leq \sigma_{2} \leq \frac{1}{2}\right) \\ \tau^{\frac{1}{3}-\frac{1}{3} \sigma_{2}} \log \tau & \left(\frac{1}{2}<\sigma_{2}<1\right) .\end{cases}
$$

Collecting these estimates, we obtain that

$$
\sum_{j} U\left(s_{1}, s_{2} ; 2^{-j} \tau\right) \ll \begin{cases}\tau^{1-\frac{2}{3}\left(\sigma_{1}+\sigma_{2}\right)} \log ^{2} \tau & \left(0 \leq \sigma_{1} \leq \frac{1}{2}, 0 \leq \sigma_{2} \leq \frac{1}{2}\right) \\ \tau^{\frac{5}{6}-\frac{1}{3}\left(\sigma_{1}+2 \sigma_{2}\right)} \log ^{3} \tau & \left(\frac{1}{2}<\sigma_{1}<1,0 \leq \sigma_{2} \leq \frac{1}{2}\right) \\ \tau^{\frac{5}{6}-\frac{1}{3}\left(2 \sigma_{1}+\sigma_{2}\right)} \log ^{3} \tau & \left(0 \leq \sigma_{1} \leq \frac{1}{2}, \frac{1}{2}<\sigma_{2}<1\right) \\ \tau^{\frac{2}{3}-\frac{1}{3}\left(\sigma_{1}+\sigma_{2}\right)} \log ^{4} \tau & \left(\frac{1}{2}<\sigma_{1}<1, \frac{1}{2}<\sigma_{2}<1\right) .\end{cases}
$$

From (4.7), (4.18) and (4.19), we get the assertion (1.8).

\section{References}

[1] S. Akiyama, S. Egami and Y. Tanigawa, An analytic continuation of multiple zetafunctions and their values at non-positive integers, Acta Arith. 98 (2001), 107-116.

[2] S. AKIYAMA and Y. TANigAWA, Multiple zeta values at non-positive integers, Ramanujan J. 5 (2001), 327-351.

[3] F. V. AtKinson, The mean-value of the Riemann zeta-function, Acta Math. 81 (1949), 353-376.

[4] A. Erdélyi, W. Magnus, F. Oberhettinger and F. G. Tricomi, "Higher Transcendental Functions I", McGraw-Hill, New-York, 1953.

[5] I. S. Gradshteyn and I. M. Ryzhik, "Tables of Integrals, Series and Products", Academic Press, Inc., 1979. 
[6] S. W. Graham and G. Kolesnik, "Van der Corput's methods of exponential sums", London Math. Society, LNS Vol. 126, Cambridge University Press 1991.

[7] J. L. H. HAFNER, New omega results in a weighted divisor problem, J. Number Theory 28 (1988), 240-257.

[8] M. N. HuXley, "Area, Lattice Points and Exponential Sums", Oxford Science Publications, Clarendon Press, Oxford, 1996.

[9] M. N. HuXLEY, Integer points, exponential sums and the Riemann zeta function, In: "Number Theory for the Millennium, Proc. Millennial Conf. Number Theory", Vol. II, M. A. Bennett et al. (eds.), A K Peters 2002, 275-290.

[10] H. IshiKaWA and K. MATSUmoto, On the estimation of the order of Euler-Zagier multiple zeta-functions, Illinois J. Math. 47 (2003), 1151-1166.

[11] A. IVIĆ, "The Riemann Zeta-Function", John Wiley \& Sons, 1985.

[12] E. KRÄTZEL, "Lattice Points", Kluwer Academic Publishers, 1988.

[13] K. Matsumoto, On the analytic continuation of various multiple zeta-functions, In: "Number Theory for the Millennium, Proc. Millennial Conf. Number Theory", Vol. II, M. A. Bennett et al. (eds.), A K Peters 2002, 417-440.

[14] Y. OHNo, A generalization of the duality and sum formulas on the multiple zeta values, J. Number Theory 74 (1999), 39-43.

[15] E. C. Titchmarsh, On Epstein's zeta-function, Proc. London Math. Soc. (2) 36 (1934), 485-500.

[16] E. C. Titchmarsh, "The Theory of the Riemann Zeta-Function" (Revised by D. R. Heath-Brown), Clarendon press Oxford, 1986.

[17] J. Q. ZhaO, Analytic continuation of multiple zeta function, Proc. Amer. Math. Soc. 128 (2000), 1275-1283.

Department of Mathematical Sciences,

Faculty of Science,

Yamaguchi University,

Yoshida 1677-1 Yamaguchi 753-8512, Japan

kiuchi@yamaguchi-u.ac.jp

Graduate School of Mathematics,

Nagoya University,

Nagoya, 464-8602, Japan

tanigawa@math.nagoya-u.ac.jp 